\title{
EFECTO DE LA RAZA, PESO DE SACRIFICIO Y SEXO EN EL QUINTO CUARTO EN RAZAS OVINAS CANARIAS
}

\author{
THE EFFECT OF BREED, SLAUGHTER WEIGHT AND SEX IN FIFTH-QUARTER IN \\ CANARY SHEEP BREEDS
}

\author{
Pérez, V. ${ }^{1 *}$; Camacho, A. ${ }^{1}$; Mata, J. ${ }^{1}$ y Bermejo, L. ${ }^{1}$
}

\begin{abstract}
${ }^{1}$ Area de Producción Animal. Departamento de Ingeniería, Producción y Economía Agraria. Universidad de La Laguna. La Laguna. Tenerife. *vperezroja@gmail.com
\end{abstract}

\section{Palabras clave adicionales}

Despojos. Razas locales. Vísceras.

\section{RESUMEN}

Se estudiaron 60 corderos de raza Canaria y 60 de raza Canaria de Pelo, mitad machos y mitad hembras, sacrificados a $9,78 \pm 0,49 \mathrm{~kg} ; 15,8 \pm 0,66$ $\mathrm{kg}$ y $24,9 \pm 0,76 \mathrm{~kg}$. El objetivo del trabajo fue conocer el efecto de la raza, sexo y peso de sacrificio sobre la importancia porcentual del quinto cuarto y sus componentes. El peso total del quinto cuarto fue mayor en la raza Canaria, aunque su porcentaje de órganos comestibles, fue inferior al de la raza Canaria de Pelo. Los intestinos, la piel y la cabeza fueron las partes que mayor peso porcentual tuvieron. Al aumentar el peso de sacrificio, la importancia del quinto cuarto se redujo debido al menor porcentaje de cabeza y autópodos. En los machos, la cabeza, vejiga y autópodos, alcanzaron mayor proporción y en las hembras, la piel. El porcentaje de piel incrementó al aumentar el peso de sacrificio sólo en los corderos de lana.

\section{SUMMARY}

Sixty Canaria lambs and 60 Canaria Hair breed lambs (half male and half female), slaughtered to $9.78 \pm 0.49,15.8 \pm 0.66$ and $24.9 \pm 0.76 \mathrm{~kg}$ live weight were studied. The objective of study was to determine the effect of breed, slaughter weight and sex of lamb on percentages of fifth quarter and their components. Canaria breed lambs showed a higher weight for fifth quarter, and its percentage of edible organs was lower than in Canaria Hair breed. The intestines, skin and head were the parts that had greater weight percentage.

\author{
AdDitiOnAL KEYWORDS
}

Local breeds. Offal. Viscera.

The importance of the fifth quarter was reduced when slaughter weight increased due to lower percentage of head and legs. Males showed higher values for head, legs and bladder; females showed higher values for the skin. Skin percentage increased when increasing the slaughter weight only in wool lambs.

\section{INTRODUCCIÓN}

El quinto cuarto es el conjunto de despojos o menudos, vísceras y subproductos obtenidos tras el sacrificio de los animales de abasto (Delfa, 1992), actuando como factores de variación del mismo, la especie, genotipo, sexo, edad y estado sanitario del animal (Joy et al., 2005). El valor porcentual del quinto cuarto influye en el rendimiento comercial de la canal, y por tanto en su valor económico (Delfa et al., 2005). Parte del quinto cuarto puede comercializarse permitiendo ingresos complementarios. Se considera que el valor comercial del quinto cuarto en ganado ovino representa un $15,9 \%$ del precio de venta de la canal (Delfa et al., 2005).

El Instituto Canario de Investigaciones Agrarias (ICIA), y las dos Universidades Canarias, estudian desde 2006, a través de un proyecto INIA, la calidad cárnica de dos razas autóctonas de protección especial 
(R.D. 1682/1997): la raza Canaria (lana) y la raza Canaria de Pelo. Estas razas, junto a la raza Palmera (lana), son la base de la producción local de carne de ovino (1591 t, según ISTAC, 2010). El objetivo de este trabajo, es analizar el efecto de la raza, peso de sacrificio y sexo de corderos sobre la importancia porcentual del quinto cuarto y sus diferentes componentes en las razas Canaria y Canaria de Pelo.

\section{MATERIAL YMÉTODOS}

Este trabajo cumplió con las normas del Comité de Investigación Ética y Confort Animal de la Universidad de la Laguna (R.D. Ley 14/2007).

Se estudiaron 120 corderos, 60 de la raza Canaria de Pelo y 60 de la raza Canaria. Para cada raza se establecieron tres pesos de sacrificio $(9,78 \pm 0,49 \mathrm{~kg}, 15,8 \pm 0,66 \mathrm{~kg}$ y $24,9 \pm 0,76 \mathrm{~kg}$ ), estudiando para cada peso 10 machos y 10 hembras. Todos los corderos nacieron y se criaron en una explotación ovina intensiva (Gorón Súr S.L.), ubicada al sur de la isla de Tenerife, (Las GalletasArona), a unos $200 \mathrm{~m}$ sobre el nivel del mar. Se trata de una zona (Cabildo de Tenerife, 2011) de clima cálido con una temperatura media anual de $20^{\circ} \mathrm{C}$ y escasas precipitaciones $(170 \mathrm{~mm} / \mathrm{año})$.

Los corderos sacrificados a 9 y $15 \mathrm{~kg}$ permanecieron con las madres, desde el nacimiento. Los sacrificados a $24 \mathrm{~kg}$ se separaron de las madres al superar los $15 \mathrm{~kg}$ de peso, siendo alojados en la zona de cebo. Para todos los corderos la lactancia fue libre. A los 15 días de vida comenzaron a recibir pienso de iniciación (0,9 UFC, $18 \%$ PB) y heno de alfalfa, y a los 40 días se cambió a un pienso de cebo (0,9 UFC, $16 \% \mathrm{~PB})$.

Cuando los corderos alcanzaban el peso de sacrificio, eran trasladados al matadero insular. Tras un periodo de ayuno de 12 horas, los animales fueron pesados y sacrificados. En todas las canales fueron pesadas las siguientes vísceras y despojos: bazo, cabeza, patas, piel, órganos sexuales, timo, corazón, hígado, pulmones-tráquea, intestino vacío y vejiga. Se determinó la proporción de cada una de estas partes en relación al peso de sacrificio. La suma del hígado, pulmones-tráquea y corazón constituyen la proporción de órganos comestible.

Los datos se analizaron con el programa estadístico SPSS 15.0 (SPSS Inc., 2006). Las diferencias entre grupos fueron determinadas con el test de Scheffé.

\section{RESULTADOSYDISCUSIÓN}

El efecto de la raza, peso de sacrificio y sexo en los componentes del quinto cuarto se recogen en la tabla $\mathbf{I}$.

La raza Canaria mostró mayor proporción del quinto cuarto y menor de órganos comestibles que la raza Canaria de Pelo $(p<0,001)$. Fundamentalmente los mayores porcentajes de piel $(\mathrm{p} \leq 0,001)$ y cabeza $(\mathrm{p} \leq 0,001)$ de la raza Canaria, originaron las diferencias raciales en el porcentaje total del quinto cuarto, lo que podría estar relacionado con la morfología y precocidad de las razas. La piel es considerada un órgano de desarrollo precoz (Atti et al., 2003; Mahouachi y Atti, 2005), al igual que la cabeza (Hammond, 1966). La menor proporción de la raza Canaria en el porcentaje de órganos comestibles se debió a la menor proporción de corazón $(\mathrm{p} \leq 0,01)$, hígado $(\mathrm{p} \leq 0,001)$ y pulmón-tráquea $(\mathrm{p} \leq 0,001)$.

Al aumentar el peso de sacrificio disminuyó la proporción del quinto cuarto, aunque sólo significativamente cuando se incrementaba de 10 a $16 \mathrm{~kg}$. El descenso se debió a la disminución del peso de la cabeza $(\mathrm{p}=0,001)$ y los autópodos $(\mathrm{p}=0,001)$ (tabla I), que son órganos de desarrollo precoz (Hammond, 1966). Resultados similares fueron encontraron por Cañeque et al. (1996) en corderos Talaveranos, y Díaz (2001) en corderos Manchegos.

Al aumentar el peso de sacrificio se produjo un descenso significativo en la proporción del bazo, cabeza, corazón, autópodos, pulmón-tráquea, timo y vejiga. Tam- 


\section{EL QUINTO CUARTO EN RAZAS OVINAS CANARIAS}

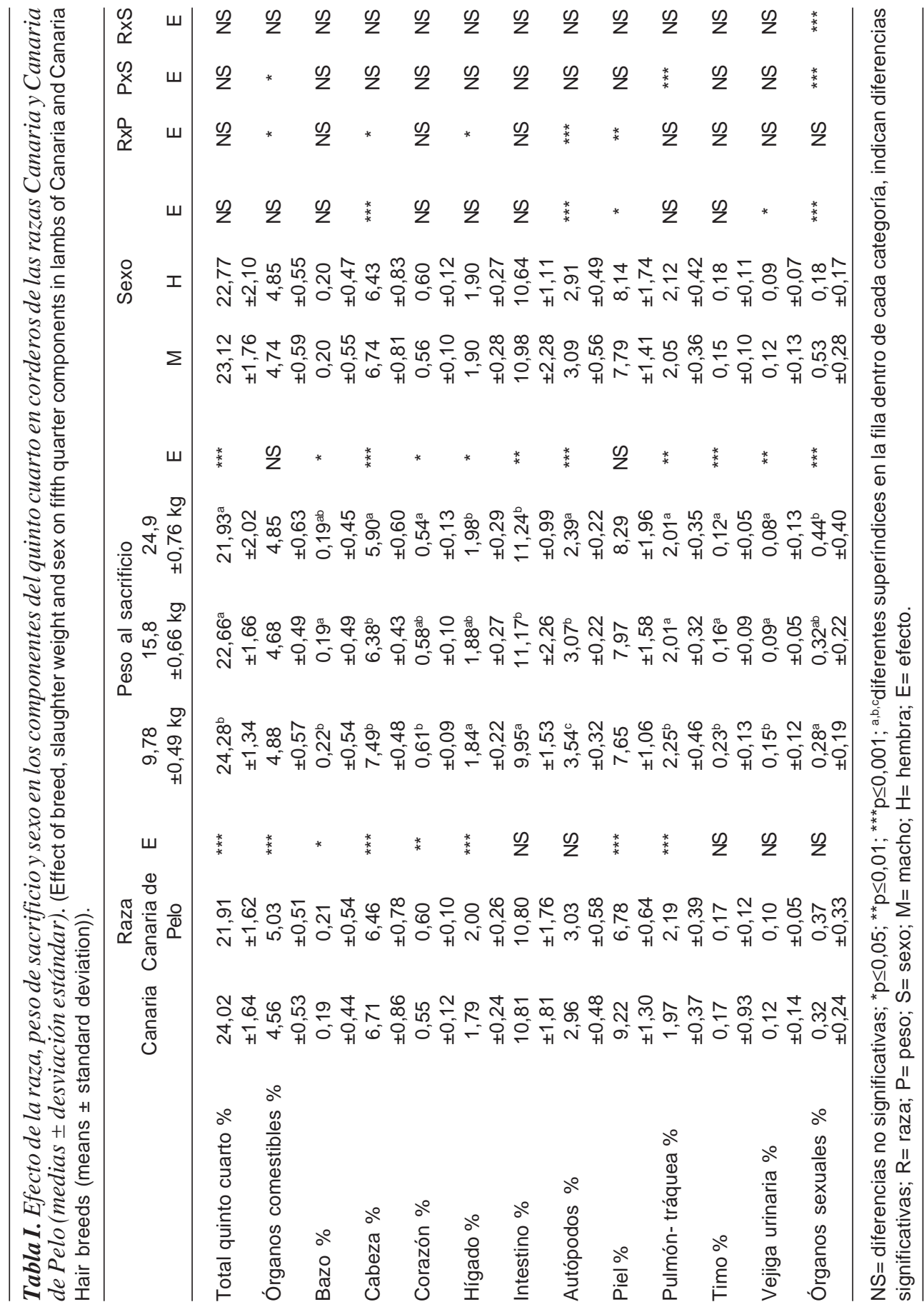

Archivos de zootecnia vol. 63, núm. 242, p. 291. 
bién se observó un descenso significativo en el peso del intestino (tabla I) pero sólo de 10 a $16 \mathrm{~kg}$. Por el contrario, el porcentaje de hígado y órganos sexuales aumentó al pasar de 10 a $16 \mathrm{~kg}(\mathrm{p} \leq 0,01)$. La mayor proporción de pulmón-tráquea $(\mathrm{p} \leq 0,01)$ en los corderos lechales, puede obedecer a la madurez temprana de estos órganos (Butterfield, 1988), resultado que coincide con lo observado por Manso et al. (1998) en la raza Churra, catalogada como precoz (Sañudo et al., 1997).

El porcentaje del quinto cuarto obtenido para los corderos de $10 \mathrm{~kg}(24,28 \%)$ es menor que el encontrado por Díaz (2001) en corderos Manchegos de igual peso $(39,9 \%)$ y Velasco et al. (2000) en corderos lechales Talaveranos (40,91\%), lo que podría deberse a diferencias en su alimentación.

El porcentaje de piel fue superior en las hembras, posiblemente debido a que son más precoces que los machos (Díaz, 2001). Los porcentajes de cabeza y autópodos fueron significativamente superiores en los machos, coincidiendo con lo encontrado por Díaz (2001) en lechales Manchegos, hecho que según el autor se debió a la diferencia de precocidad de los sexos. En este trabajo, los machos también mostraron mayor proporción de vejiga urinaria.

En la figura 1, se observa que en los corderos de pelo el porcentaje de piel permanece constante al aumentar el peso de sacrificio, mientras que en la raza de lana este porcentaje aumenta. Resultados similares fueron observados por Osório et al. (2000) en corderos de las razas Polwarth, Corriedale, Romney Marsh y Texel, donde la raza Polwarth (lana) fue la que presentó un mayor incremento en el porcentaje de piel al aumentar la edad de sacrificio, y la raza Texel (carne) el menor, resultado que, según los autores, se debió a un mayor crecimiento de la lana en la raza Polwarth. Así pues, el aumento en la proporción de piel en los corderos de lana, al aumentar el peso de sacrificio, podría deberse a un mayor crecimiento de la lana conforme crece el cordero, hecho que no se produciría en el pelo.

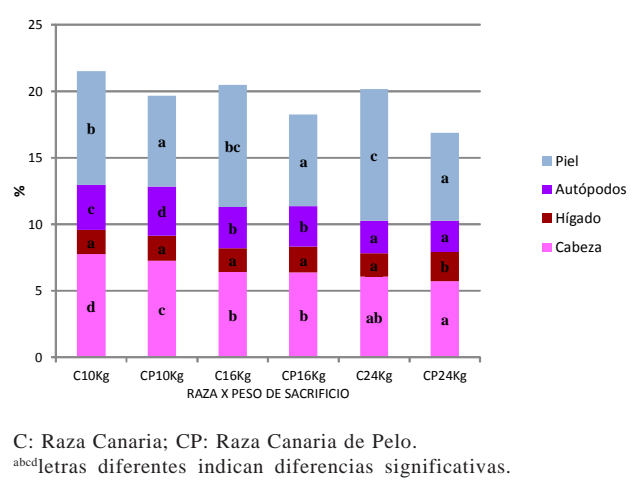

Figura 1. Componentes del quinto cuarto en la interacción raza x peso. (Components of the fifth quarter in breed $x$ weight interaction).

Los corderos de lana sacrificados a 10 $\mathrm{kg}$, mostraron mayor proporción de cabeza, los de pelo, mayor proporción de patas, y los de lana sacrificados a $24 \mathrm{~kg}$, la mayor proporción de órganos comestibles.

Se encontraron (figura 2) diferencias significativas en el porcentaje de pulmóntráquea. Estas diferencias se observaron en los corderos de $10 \mathrm{~kg}$, donde las hembras mostraron mayor proporción. Pérez et al. (2007) en corderos de $15 \mathrm{~kg}$ de la raza Suffolk Down x Merino precoz alemán no evidenciaron diferencias en proporción de pulmóntráquea en la interacción peso x sexo.

La proporción de órganos sexuales es

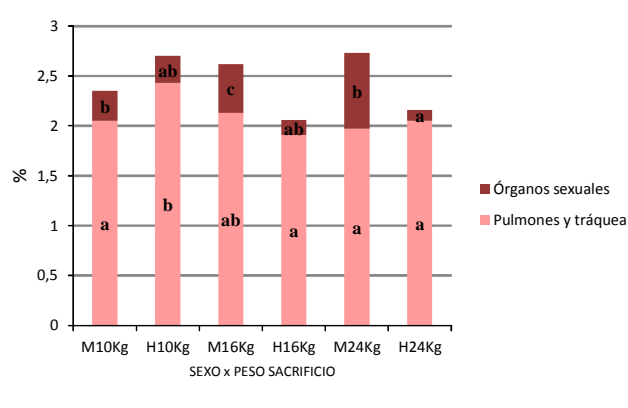

M: macho; H: hembra.

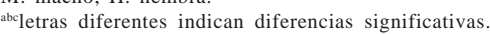

Figura 2. Componentes del quinto cuarto en la interacción sexo x peso. (Components of the fifth quarter in sex $x$ weight interaction). 


\section{EL QUINTO CUARTO EN RAZAS OVINAS CANARIAS}

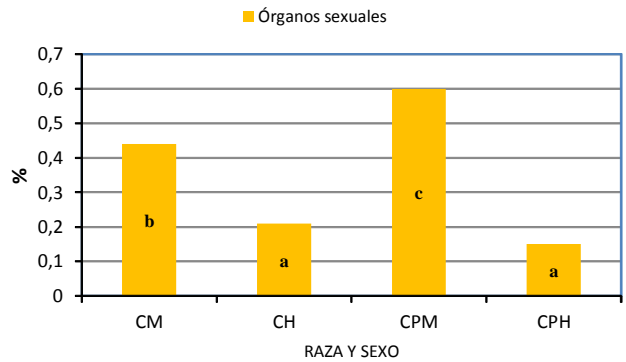

C: Raza Canaria; CP: Raza Canaria de Pelo; M: macho; H: hembra abcletras diferentes indican diferencias significativas.

Figura 3. Componentes del quinto cuarto en la interacción raza $x$ sexo. (Components of the fifth quarter in breed $x$ sex interaction).

\section{BIBLIOGRAFÍA}

Atti, N., Ben Salem, H. and Priolo, A. 2003. Effects of polyethylene glycol in concentrate or feed blocks on carcass composition and offal weight of Barbarine lambs fed Acacia cyanophylla Lindl. foliage. Anim Res, 52: 363-375.

Butterfield, R.M. 1988. New concepts of sheep growth. Department of Veterinary Anatomy Ed. University of Sidney. South Australia.

Cabildo de Tenerife. 2011. Datos meteorológicos de la estación Las Galletas. Arona. Tenerife.

Cañeque, V.; Lauzurica, S.; López, D.; Cantero, M.A.; Ruiz de Huidobro, F.; Pérez, C.; Gayán, J.; Sancha, J.L. y Velasco, S. 1996. Producción de carne en corderos lechales de raza Talaverana. I. Rendimientos en el matadero e importancia de los despojos. In: XXI Jornadas Científicas de la Sociedad Española de Ovinotecnia y Caprinotecnia. Logroño. pp. 603-609.

Delfa, R. 1992. Predicción de la composición corporal y de la canal a partir del animal vivo y de la canal. Ovis, 23: 25-56.

Delfa, R.; Tort, S.; Bergua, A.; Lahoz, F.; Revilla, R. y Joy, M. 2005. Efecto del sistema de producción sobre el quinto cuarto de corderos Churros Tensinos. XXX Jornadas Científicas y IX Internacionales de la Sociedad Española de Ovinotecnia y Caprinotecnia. Granada. pp. 55-58.

Díaz, M.T. 2001. Características de la canal de corderos lechales manchegos. Correlaciones y ecuaciones de predicción. Tesis doctoral. Universidad Complutense. Madrid. significativamente mayor en los machos de la raza Canaria de Pelo (figura 3).

\section{CONCLUSIONES}

La raza afecta a la parte comestible del quinto cuarto, no así el peso de sacrificio y el sexo en corderos de razas Canaria y Canaria de Pelo.

\section{AGRADECIMIENTOS}

Al INIA por la financiación del proyecto de investigación (RTA/2006-00174-00-00), a la granja Gorón Sur S.L. y al Matadero Insular de Tenerife.

Hammond, J. 1966. Principios de la explotación animal. Reproducción, crecimiento y herencia. Ed. Acribia. Zaragoza. España.

ISTAC. 2010. Instituto Canario de Estadística Consejería de Agricultura, Ganadería y Pesca. Islas Canarias.

Joy, M.; Tort, S.; Bergua, A.; Lahoz, F.; Revilla, R. y Delfa, R. 2005. El quinto cuarto en porcentaje. Piel, órganos comestibles e industriales de corderos Churro Tensinos criados en diferentes sistemas de producción. XXX Jornadas Científicas y IX Internacionales de la Sociedad Española de Ovinotecnia y Caprinotecnia. Granada. pp. 67-69.

Mahouachi, M. and Atti, N. 2005. Effects of restricted feeding and re-feeding of Barbarine lambs: intake, growth and non-carcass components. Anim Sci, 81: 315-312.

Manso, T.; Ruiz, A. y Castro, T. 1998. Rendimiento a la canal, quinto cuarto y despiece de corderos de raza Churra sometidos a distintas estrategias de alimentación. Arch Zootec, 47: 73-84.

R.D. Ley 14/2007. Sobre investigación biomédica. BOE núm. 159 de 4 de Julio.

Osório, J.C.; Oliveira, N.M.; Osório, M.T.; Pimentel, M. y Pouey, J.L. 2000. Efecto de la edad al sacrificio sobre la producción de carne en corderos no castrados de cuatro razas. Rev Bras Agrocien, 6: 161-166.

Pérez, P.; Maino, M.; Köbrich, C.; Morales, M.S. y Pokniak, J. 2007. Efecto del peso de sacrificio 


\section{PÉREZ, CAMACHO, MATA YBERMEJO}

y sexo sobre la canal de corderos lactantes del cruce Suffolk Down x Merino Precoz Alemán. FCV-LUZ, 17: 621-626.

R.D. 1202/2005. Sobre protección de los animales utilizados para experimentación y otros fines científicos. BOE núm. 252 de 21 octubre.

Sañudo, C.; Campo, M.M.; Sierra, I.M.; María, G.A.;

Olleta, J.L. and Santolaria, P. 1997. Breed effect on carcass and meat quality of suckling lambs.
Meat Sci, 46: 357-365

SPSS. 2006. SPSS 15.0 for Windows. SPSS. Inc. Chicago. USA.

Velasco, S.; Lauzurica, S.; Cañeque, V.; Pérez, C.; Ruiz de Huidobro, F.; Manzanares, C. and Díaz, M.T. 2000. Carcass and meat quality of Talaverana breed sucking lambs in relation to gender and slaughter weight. Anim Sci, 70: 253-263.

Archivos de zootecnia vol. 63, núm. 242, p. 294. 